\title{
A Preferences Based Approach for Better Comprehension of User Information Needs
}

\author{
Sondess Missaoui ${ }^{1(\bowtie)}$ and Rim Faiz ${ }^{2}$ \\ 1 LARODEC, ISG, University of Tunis, Bardo, Tunisia \\ sondes.missaoui@yahoo.fr \\ 2 LARODEC, IHEC Carthage University, Carthage Presidency, Carthage, Tunisia \\ Rim.Faiz@ihec.rnu.tn
}

\begin{abstract}
Within Mobile information retrieval research, context information provides an important basis for identifying and understanding user's information needs. Therefore search process can take advantage of contextual information to enhance the query and adapt search results to user's current context. However, the challenge is how to define the best contextual information to be integrated in search process. In this paper, our intention is to build a model that can identify which contextual dimensions strongly influence the outcome of the retrieval process and should therefore be in the user's focus. In order to achieve these objectives, we create a new query language model based on user's pereferences. We extend this model in order to define a relevance measure for each contextual dimension, which allow to automatically classify each dimension. This latter is used to compute the degree of change in result lists for the same query enhanced by different dimensions. Our experiments show that our measure can analyze the real user's context of up to 12000 of dimensions (related to 4000 queries). We also show experimentally the quality of the set of contextual dimensions proposed, and the interest of the measure to understand mobile user's needs and to enhance his query.
\end{abstract}

Keywords: Mobile search - User's context - Relevance - User's preferences

\section{Introduction}

The heart of Mobile Information Retrieval (IR) research is how to exploit environmental and personal information at different contextual dimensions so that users receive relevant results quickly and conveniently. Regarding Mobile IR, context provides an important basis for identifying and understanding user's query. The representative information about the user's context should enable IR systems to better meet information needs. This domain is in rise according to many studies such as $[5,9,16,18]$, and this often caused by the huge development and processing in mobile devices technologies. Those later have special 
characteristics which make them very advanced to sense different user's contextual dimensions. In fact, PDAs or smartphones are designed to take into account the user's situation and can ultimately allow the inference of the image or video content from the context using GPS, Bluetooth and even more sensors. On the other hand unlike the desktop systems, mobile devices usually have small screen size and tiny keypads and much more problems such as disconnected operation, low processing speed.

Beyond the special characteristics of mobile devices, people want to access information anytime and anywhere with the Smartphones they carry all the time. Within this practise, mobile information content as well as effective mobile search systems has attracted increasing attention. This suggests a latent demand for mobile IR that is able to enhance results visualization through the knowledge of the user's context and specificities. For this purpose, IR systems must take into account considerable challenges such as the users requirement (when they need information about something, they expect to get it right away), the mismatch query problem [14], the dynamic and less predictable environments, the shorter sessions compared with desktop usage. According to [9], users describe their needs in shorter query and use less number of queries by session. They usually consult only the first page of results [20].

Now faced with this evolving mobile technologies, exploiting the user context to enhance search quality became a necessity. For example, taping the query "Jobs" a user can search for a summer job, a permanent job, a job around his location (in his city), a job according to his interest (preferences) or even a statistical review about the number of job opportunities created in his country. So, for a clear distinction of the user's need we have to integrate different contextual dimensions. Often, with mobile applications, some aspects of the user's context are available, and this context can affect what sort of information is relevant to the user. The context can include a wide range of dimensions that characterize the situation of the user. But the question arises what is the impact of each contextual dimension on the quality of results? and How it can be considered as relevant to enhance the search task? What contextual dimensions reflect better the mobile user's need and lead to the appropriate search results? Our work consists in searching a new metric of dimensions impact on the search results quality.

In fact, the mobile users enter a limited number of terms in a query. This creates a big challenge to the IR systems which called "query mismatch problem" [14]. So many studies integrate different context fields to enhance the query such as [18], and especially to model the context, allowing to identify information that can be usefully exploited to improve search results such as $[4,5,8,31]$ and [21].

In this paper, we focus our research efforts in this area that has received less attention which is the context filtering. We have brought a new approach that has addressed this issue. How to define the relevant contextual dimension accurately and rapidly? Our work has proceeded in terms of adapting the mobile context to user's preferences and identifying relevant contextual dimensions. 
We propose a new approach allows to define the most relevant and influential user's context dimensions for each search situation.

In fact, our hypothesis is that an accurate and relevant contextual dimension is the one that provides an interesting improvement in the Preference query profile. Those dimensions can improve the quality of search by proposing to the user results tailored to his current situation and preferences.

The remainder of this paper is organized as follows. In Sect. 2, we give a comparison between Mobile IR and Traditional IR, then we precise the specificities of the emerging area of mobile search. In Sect. 3 we give an overview of related work which address Context-centred mobile web search. We describe in Sect. 4, the Context adaptation approach to user's preference. In Sect. 5, we discuss experiments and obtained results. Finally, Sect. 6 concludes this paper and outlines future work.

\section{Mobile Information Retrieval VS Traditional Information Retrieval}

Mobile Information Retrieval and Traditional Information Retrieval approaches pursued the same aim, which is to return relevant response to meet an information need from a collection of documents, but differ in their responses and the means implemented. Their fundamental difference is the nature of the information they return.

Traditional IR approaches consider that user needs are described fully by the user query. These approaches provide the same results for the same keyword queries even though these latter are submitted by different users in different contexts. Whereas, Mobile IR approaches aim to tailor search results to individual users by taking into account their intentions, preferences and dynamic physical context. These approaches aim to satisfy the information needs of a mobile user retrieving information via Smartphones.

In fact, Mobile IR is an expanding area, which take advantage from the growing diversity of Smartphone as well as the availability of a large amount of mobile media content that have been generated rapidly.

Successfully, a key distinction of Mobile search systems, makes them more advanced than their traditional counterparts, is that the user's environment is dynamic, in contrast to information searching in the desktop, where a user's environment is less likely to change. The identification of a such user's environment allows to identify information that can be usefully exploited to the aim of improving search effectiveness. Actually, by user's context we refer to the information characterizing the user's physical and social context. The contextual information may include geographic data, Time, activity, preferences of the user, etc. In fact, Smartphones have advanced features that make them able to generate such information and a more powerful understanding of the user's current context (situation). Recent advances in mobile telecommunications technology have seen the functionality of phones evolve from predominantly voice exchange to the capacity to process and gathered contextual and personal information. 
Traditional IR systems that assume a stagnant user's environment are no longer suitable for such extremely mobile scenarios.

\subsection{Specificities of Mobile Information Retrieval}

Indeed, in mobile environment many factors bring together to present challenges issues to the IR systems. Challenges are of changing location and social context, restricted time for information access, and the need to adapt resources with concurrent physical characteristics of mobile devices. Those latter can play a main role in the paradigm of mobile search. Hence, the use of mobile devices influences not only the types of information people seek but also the ways they attempt to access. The impact of Smartphones, and mobile devices in general, in the search process is related to their specificities and characteristics. As they have more features than their computer counterparts, Smaetphones can provide efficient information where the relevant data that characterizes the user context is represented (such as location, weather, time, social networks and so on).

The availability of a context model may enhance the search process and allows to personalize it by considering several dimensions in the relevance assessment process. But at this point obvious questions arise: (1) how to make this contextual information available to an IR system? (2) how to model it? (3) and how to exploit the user context accurately in the search process?

Independently of the context model, an interesting aspect which emerges in mobile IR is that the availability of a new mobile technologies makes it necessary to process large amounts of data on small screens and in power-limited settings, which is a Content adaptation issue. Actually Mobile IR interprets both the content and the context to extract useful information and relationships, in order to proactively improve designs for mobile phones and related devices $[9,16]$. To this aim, two important issues that have been addressed in the literature related to Mobile IR concern Context-awareness and Content Adaptation. In this paper, we discuss the Context-awareness issue which is highly related to our research.

In the next part (cf. Section 3), we will discuss some recent research efforts, that have addressed the problem of personalizing search using user context.

\subsection{Discussion}

Traditional IR approaches are efficient to find relevant information with very high precision when knowing the right words to use in a search query. These approaches consider that user needs are described fully by the user query, which isn't always true in a mobile search environment.

In fact, Traditional IR approach can't provide personalized results accurate to the mobile user situation and intention when it considers the query as the main clue that specifies the user information need. Although the advances in mobile technology, Smartphones still have smaller screens, less processing power and low memory, those factors influence mobile users seeking behaviour. Since, mobile individuals become less patient and typically use one or two keywords maximum per web search and their queries shorter and more ambiguous. Those 
seeking habits create the query mismatch problem [14], which cannot be solved using Traditional IR approaches.

Indeed, Traditional approaches haven't the potential to enhance the user query with the user profile, interests, preferences or context that could be explicitly set by the user or gathered implicitly from the user search history. Thereby, an emerging need, of scalable algorithms that can perform well in power-limited settings, appeared. Hence, research efforts focus on efficient ways to process large amounts of data and to personalize the mobile search process.

Mobile IR is a significant task in information retrieval and when coupled with context awareness technologies they can become key tools for Mobile search applications. Context-aware computing is an interesting paradigm in which Mobile IR can take advantage of contextual techniques and algorithms to produce a search outcome in response to a user's information need, which is tailored to the specific context. In the next section we introduce and discuss Contextcentred approaches for Mobile IR.

\section{Context-Centred Mobile Information Retrieval}

In recent years there has been an increasing research interest in the problem of contextualizing mobile search. Furthermore, with the availability of mobile devices and technologies that can detect the user's context, Context-centred Mobile IR are applied to produce a search outcome in response to a mobile user's query. In fact, exploiting the user context, become necessary to define any process aimed at tailoring user's information need and enhancing mobile search quality.

To discuss this paradigm, we present, in the following, the key notion of context. Then, we discuss some approaches proposed in the literature to exploit the user context and usefully apply it to the aim of improving search effectiveness.

\subsection{Context-Awareness}

Context-awareness is an expanding and vital field of research and numerous definitions of context exist because of the multidisciplinary and rich nature of the topic. There are many research efforts related to context-aware paradigm, studies from various points of view such as Schilit et al. [2], they define context as "where you are, who you are with, and what resources are nearby". This might suggest that context is more focused on the user's surrounding as opposed to his inner states. Morse et al. [1], describe context as "implicit situational information". As chosen one, we opt for the definition of Dey and Abowd [1], in which the context is: "Any information that can be used to characterize the situation of an entity. An entity is a person, place, or object that is considered relevant to the interaction between a user and an application, including the user and applications themselves. In this definition context result in relations between applications, situations, and entities." 
In fact, in context-aware sensitive mobile search, approaches focuse on the aim of modelling the user's current context, and exploit it in the retrieval process. Given the specificity of Mobile devices, looking for information is facing several challenges such as recognizing the user's intention behind the query (Bouidghaghen et al. [18]), personalizing web search (Tsai et al. [5], Pitkow et al. [10], Ahn et al. [8]), enhancing the user's query with the current context to better meet the individual needs [9], understanding the nature of research practices [16], and Modelling the context $([4,19,21,29])$. In fact, mobile users are often engaged in a specific task in the real world while they are moving, since their context is in a state of change, which adds further complexity. With this in mind, Context-aware approaches should use the right information about the user's current context as a means to deliver relevant content to that situation, within the purpose to build mobile search systems that consider increasing the precision of results.

In the literature several approaches have been proposed, which can be roughly categorized into three main classes:

- One dimension fits all approaches

- Approaches use a predefined set of contextual dimensions

- Context adaptation approaches

\subsection{One Dimension Fits All Approaches}

Regarding contextual information, some approaches which are characterized as "one dimension fits all" using one same contextual dimension to personalize all search queries regardless the query's keyword or the user's intentions behind it. These approaches consider user's context as one dimension in all search sessions. In this category, location is probably the most commonly used variable in context recognition. Several studies such as Bouidghaghen et al. [18], Welch and Cho [15], Chirita et al. [19], Vadrevu et al. [30] and Gravano et al. [13] have built models able to categorize queries according to their geographic intent.

Indeed, they identify the query sensitivity to location in order to determine whether the user's need is related to his geographical location or not. With the aim to personalize the search results using geographic dimension, many studies (Welch and Cho [15], Vadrevu et al. [30] and Gravano et al. [13]) have suggested to use the classification techniques to personalize the search results using current location. Location can be considered as an important context dimension but in this field it is not the only one, others can be taken into account. Some queries have no intent for localization (e.g. Microsoft office version, Horoscope) but they are "Time" sensitive.

With the aim of recognizing the user's intention behind the search, using a unique predefining context's dimension is not accurate. For example, when a mobile user is a passenger at the airport and he is late for check-in, the relevant information often depends on more than time or localization. It is a complex searching task. So, it needs some additional context dimensions such as activities and dates (e.g., flight number inferred from the user's personal calendar or 
numeric agenda). As another example let us consider a group of users are preparing for an outing with friends for the weekend. If the query "musical event" is formulated by one of them, the query evaluation should produce different contextual dimensions such as location, time and preferences.

\subsection{Approaches Use a Predefined Set of Contextual Dimensions}

Beyond the "one dimension fits all" approaches, Coppola et al. [20] and Castelli et al. [7] propose to use 'Here' and 'now' as the main important dimensions to get just incremental enhancements of existing retrieved results. Independently of the user's intention and preferences, this category of approaches relies on a predefined set of contextual dimensions for all queries and do not offer any context adaptation models to the specific goals of the users.

Several works (Gross and Klemke [32] and Aréchiga et al. [4]) operate including Time and Location besides others dimensions, most of them build a model of context which makes it possible to consider several new dimensions in the relevance assessment process such as demographic and social context. In Mymose system, Aréchiga et al. [23] propose a multidimensional context model, which includes four main dimensions (Spatial, Temporal, Task and Personal model). Those dimensions are supported by ontologies and thesaurus to represent the knowledge required for the system.

\subsection{Context Adaptation Approaches}

Actually, the quality of the personalization process is strongly related to the quality of the context's model. In fact, while all aspects of the operational mobile environment have the potential to influence the outcome search results, only a subset is actually relevant. For such reason, Some other researchers such as $[3,12]$ try to identify the appropriate contextual information in order to better meet the specific user's demand. Kessler [3] approach proposes a cognitively plausible dissimilarity measure "DIR", to automatically identify relevant contextual information. This approach is based on the comparison of result rankings stemming from the same query posed in different contexts. Such measure aims to calculate the effects of contextual changes in the IR results. Another research effort, Stefanidis et al. [12], specify context as a set of multidimensional attributes. They identify user's preferences in terms of their ability to tailor with the context state of a query.

We find that most approaches for Context-aware information delivery within Mobile IR take into account all possible gathered contextual dimensions to narrow the search. As each dimension has a unique and different impact on the retrieved results, a big challenge appeared which is about selecting the best contextual information that may help to finally retrieve highly relevant information. With this in mind, we introduce in the next section our approach for context filtering that aims to effectively identify those contextual dimensions which are eligible to encompass the user's intention and preferences. 


\section{Context Adaptation to Preferences: CAP Approach}

In this section, we focus our efforts on evaluating the user's context, in order to leave only a subset of relevant contextual dimensions. These, which go with the user's preferences and are able to enhance the search process.

\subsection{Context Model}

Context is multifaceted concept that has been studied across different research disciplines. Within Mobile IR, the context is used to overcome the limitations of the mismatch query problem [14] as well as personalization aim.

The special features of Smartphone make, location information, Time, friends contacts, and almost other user's information more accessible. All this information can be considered as context dimensions and then be used as additional features to create an efficient representation of the user's situation. In our work, the context is modelled through a finite set of special purpose attributes, called context dimensions $c_{i}$, where $c_{i} \in C$ and $C$ is a set of $\mathrm{n}$ dimensions $\left\{c_{1}, c_{2} \ldots, c_{n}\right\}$ For instance we adopt a context model that represents the user's context by only three dimensions Location, Time, Activity.

- User's physical context represented by his geographic position (Location),

- User's environmental context represented by the moment of the query submission (Time),

- And the user's organizational context (Activity).

The Fig. 1 shows the concept of user's context $C$ with an instance of a finite set of contextual dimensions Location, Time, Activity.

User's Current Context. We consider the user's current context as the user's physical, environmental and organizational situations at the moment of search.

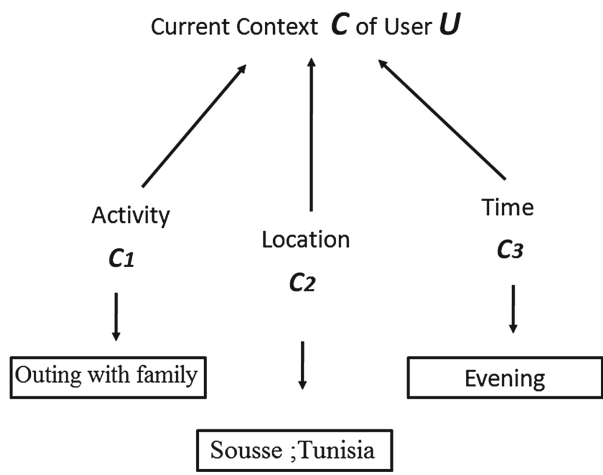

Fig. 1. An instance of a user's current context. 
It can be considered as the current state at the time of the query submission. For example, when a query such as "Restaurant" is formulated by a parent, his current situation can be defined as Location: Sousse - Tunisia; Time: Evening12/09/2012; Activity: Outing with family.

However, gathering context information is outside the scope of our research effort and our assumption is that the values of a dimension $p_{i}$ can change from a search situation to another. Contextual dimension, will be defined by computing their capacity to enhance the type of retrieved documents. We evaluate their capacity to enhance the query in order to generate results with respect to the user's preferences (Preference Query Profile). In this section, we will describe our filtering model including the main features that allow to filter the user's current context and specify the most relevant contextual dimensions to narrow the search.

\subsection{Preferences Model}

Some recent papers have investigated language modelling approach to define the user's intention behind the query. In our work we use the language modelling approach as described in [11] to filter the context. We offer a new query language model.

1. We build a language model based preferences in our research [28]. For each user's preference, we estimate a distribution of terms associated with the user's preference. Then, we can estimate the probability that a query was issued from a given preference by sampling from the term distribution of that preference.

2. We use the query preference profile to measure the relevance of a contextual dimension.

Preferences Query Profile. According to [24]: "One way to analyze a query is to look at the type of documents it retrieves". On the basis of this rule, we infer that the best way to analyze a context dimension is to look at its effect on the query. So, its effect on the type of documents the query retrieves. Specifically, it can be accomplished by examining the top $\mathrm{N}$ documents of retrieval results. The context dimensions can then be ranked by the probability that they "generated" best results after being integrated in the search process. In language model approach [33] define the document likelihood of having generated the query formally as presented by the following equations:

$$
P(Q \backslash D)=\prod_{w \in Q} P(w \backslash D)^{q_{w}}
$$

Given a query $\mathrm{Q}$ and a document $\mathrm{D}, q_{w}$ is the number of times the word w occurs in query Q. According to Croft and Lafferty [35], document language models $P(w \backslash D)$, are estimated using the words in the document. We use this ranking to build a new query language model, $P(\operatorname{Pre} \backslash Q)$, out of the top $\mathrm{N}$ documents. It 
is a new query feature in language model called "Preferences Query Profile" that helps us to define the effectiveness of the query to overcome the user's interests.

This query language model is named "the Preference profile of the query Q". In fact, a relevant retrieved result is a ranking list which meets, in a better way, the individual user needs according to their preferences. In this same spirit of thinking, we are interested in describing the personalized nature of a query. That's mean the effectiveness of the query to overcome the user's interests when it retrieving a precise topic. E.g., Searching for "Music", the mobile search system must take into account the user's preference "Jazz".

Therefore, we build a preference query profile where documents can be ranked by the probability that they have been generated depending on the user's preferences. More concretely, given a set of preferences "Pre", and a query Q, our goal is to rank the user's preferences by $\operatorname{P}(\operatorname{Pre} \backslash Q)$ which is initially defined as:

$$
\hat{P}(\operatorname{Pre} \backslash Q)=\sum_{D \in R} \hat{P}(\operatorname{Pre} \backslash D) \frac{P(Q \backslash D)}{\sum_{D \prime \in R} P(Q \backslash D)}
$$

Where $\mathrm{R}$ is the top $\mathrm{N}$ ranked document in a search result list and "Pre" is the name of the user's preference. It's a term that describes a user preference category from a database containing all user's interest (his profile). For example if a user is interested by "Sport" a set of terms such as (Football, Tennis, Baseball,...) are defined as "Pre".

$$
P(\text { Pre } \backslash D)=\left\{\begin{array}{l}
1 \text { if Pre } \in \text { Pre }_{D} \\
0 \quad \text { Otherwise }
\end{array}\right.
$$

Where $\operatorname{Pre}_{D}$ is the set of categories names of interests contained in document D (e.g. Sport, Music, News, Cinema, Horoscope ....). The profile, that describes the user's interests and preferences could be explicitly set by the user or gathered implicitly from the user search history. In our experiments, a profile is collected explicitly before starting the search session.

A very helpful step is about smoothing maximum likelihood models such as $\hat{P}\left(\operatorname{Pre} \backslash Q_{i n}\right)$. We used Jelinek-Mercer process created by [6] for smoothing. We use the distribution of the initial query $Q_{i n}$ (reference-model) over preferences as a background model.

Such background smoothing is often helpful to handle potential irregularities in the collection distribution over preferences. Also, it replaces zero probability events with a very small probability. Our aim is to assign a very small likelihood of a topic where we have no explicit evidence. This reference-model is defined by:

$$
\hat{P}\left(\operatorname{Pre} \backslash Q_{i n}\right)=\frac{1}{|N|} \sum_{D} \hat{P}(\operatorname{Pre} \backslash D)
$$

Our estimation can then be linearly interpolated with this reference model such that:

$$
P^{\prime}(\operatorname{Pre} \backslash Q)=\lambda \hat{P}(\operatorname{Pre} \backslash Q)+(1-\lambda) \hat{P}\left(\operatorname{Pre} \backslash Q_{i n}\right)
$$

Given $\lambda$ as a smoothing parameter. 
The assumption of the Preference Profile analysis is that irrelevant contextual dimension's can't improve the 'Preference Query Profile'. In fact, When we integrate an irrelevant dimension into search process, the 'Preference Query Profile' shows no variance comparing to the initial one. Given that, this contextual dimension is not important for a query and shouldn't be selected. In contrast, a relevant dimension provides a 'Preference Query Profile' with at least one peak.

Figure 2 shows an example for the effect of dimension on the Preference Language Model (preferences profile) of the query "Olympic sports". For our experiments, we choose as dimensions Time. In this example the initial query presents a flat preferences profile, while Time based profile has distinctive peaks spread over many user's preferences. Looking in depth at this graph of Fig. 2, we can see the difference between calculated preferences profile for the initial query $\left.P\left(\operatorname{Pre} \mid Q_{i n}\right)\right)$ and preference profile for the query enhanced by a contextual dimension $c_{i}$ is "Time" $P\left(\operatorname{Pre} \mid Q_{\text {Time }}\right)$. Indeed, Time dimension makes a clear improvement for some preferences (Results, Taekwando) over the other profile. But for some others user's preferences $P\left(\right.$ Pre $\left.\mid Q_{\text {Time }}\right)$ is less or equal to $\left.P\left(\operatorname{Pre} \mid Q_{i n}\right)\right)$.

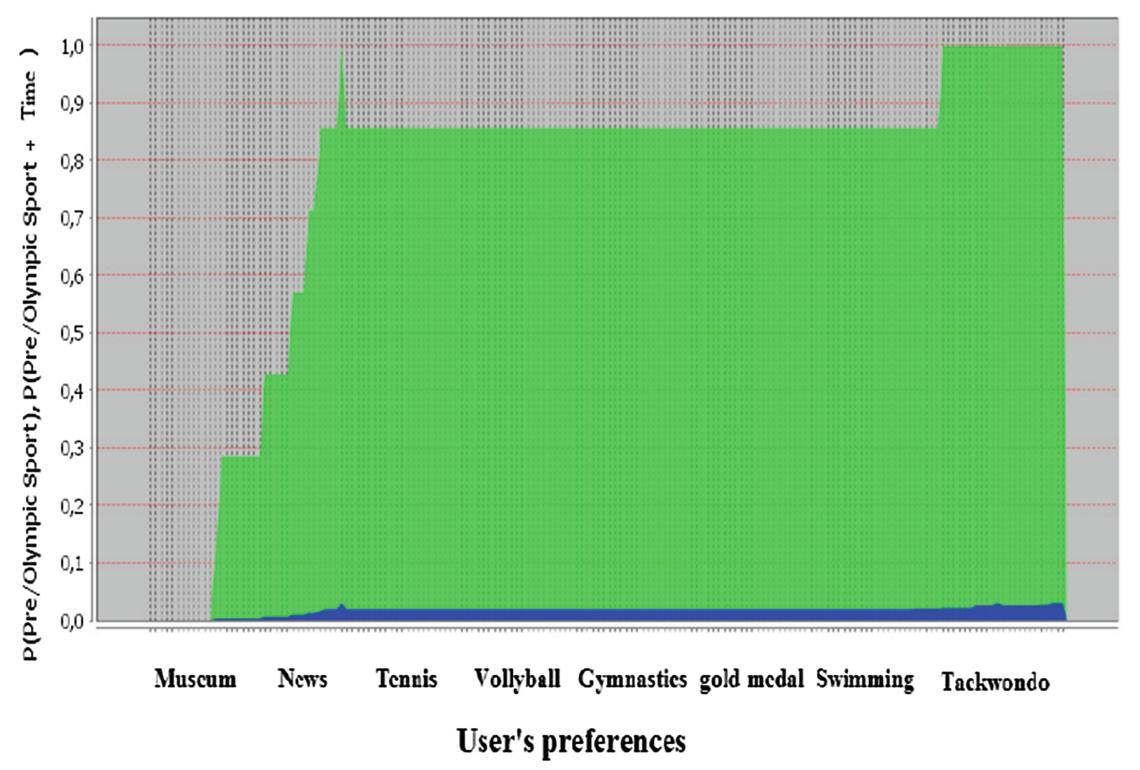

- Preference Query Profile $\quad$ Preference Profile for Query+ Time

Fig. 2. Comparison between the preferences profiles of the query "Olympic sports", and enhanced queries using the contextual dimension time.

Therefore, to define the general effect of a dimension on the search process, we need a measure to specify the relevance of each dimension according to its effect on the search outcomes. In the following, we present this measure. 


\subsection{Preference Score Measure}

Our principal objective is to adapt the user's context to his preferences automatically by filtering it. For this purpose, we need to define the most and least influential mobile context dimensions. Indeed, there is no existing measurement method that allows the quantification of the mobile contextual information pertinence especially using a statistical property of retrieved results list. Hence, the task to be accomplished is to build a relevance metric measure for contextual dimension. Our metric measure is based KL divergence [27] as an essential component to build this metric. We chose Kullback-Leibler divergence as a divergence measure issued from the domain of probability theory. It will be introduced in our approach to define the influence of each dimension on the search results. The KL divergence gives us a test of similarity to the preferences background model P $\left(\right.$ Pre $\left.\backslash Q_{i n}\right)$. Our measure 'Preference Score' is defined basically on the comparison of two result rankings. It allows to identify whether a mobile context dimension enhancing the user query at his preferences profile. The "Preference Score" is a new metric, which allows to measure the relevance degree of each dimension.

The "Preference Score" is defined as:

$$
\text { PreferenceScore }((P, Q))=D_{k l}\left(P\left(\text { Pre } \backslash Q_{p}\right), P\left(\text { Pre } \backslash Q_{i n}\right)\right)
$$

Where $\mathrm{Q}$ is the mobile query, we denote the appearance of a dimension $c_{i}$ in a mobile context (cf. Section 4.1) as $c_{i} \in C$. Let $P\left(\operatorname{Pre} \backslash Q_{i n}\right)$ the language model of the initial query used as a background distribution. And $\operatorname{P}\left(\operatorname{Pre} \backslash Q_{c}\right)$ the language model of the enhanced query using contextual dimension $c . D_{k l}$ is a Kullback-Leibler divergence, which is initially defined as:

$$
\begin{gathered}
D_{k l}\left(P\left(\text { Pre } \mid Q_{c_{i}}\right), P\left(\text { Pre } \mid Q_{i n}\right)\right)= \\
\sum_{{\text {Pre } \in \text { Pre }_{D}} P\left(\text { Pre } \mid Q_{c_{i}}\right) \log \frac{P\left(\text { Pre } \mid Q_{c_{i}}\right)}{\operatorname{P}\left(\text { Pre } \mid Q_{i n}\right)}}
\end{gathered}
$$

The proposed context-based measurement model can be expressed in a formal manner with the use of basic elements toward mathematic interpretation that build representative values from 0 to 1 , corresponding to the intensity of dimension's relevance. Being null values indicative of non importance for that dimension (it should not be integrated in personalization of the mobile IR process). In the experiment, we will try to define the threshold that a dimension should obtain to be classified as relevant or irrelevant information. In the next section, we will evaluate the effectiveness of our metric measure 'Preference Score' to classify the contextual dimensions.

\section{Experimental Evaluation}

Our goal is to evaluate the "Preference Score" metric to predict the type of user's context dimension. 


\section{$5.1 \quad$ Dataset}

For the experiments reported in this work, we used a real-world dataset which is generated through diary study. We have chosen to use a diary study for two reasons. First, the lack of a survey popular data sets for mobile search applications. Second, the diary study allows to capture data revealing the real nature of mobile user information needs. Below we describe how we managed this diary.

Diary Study and Participants. The participants (40 male, 56 female) in our study, were required to own a mobile phone and have experience searching on mobile. They include graduate students aged from 24-37, they are members of our laboratory and other ones associated with our university. The study has released in 17 weeks, we asked participants to keep a diary for this period of all their information needs and their context (Activity, Time and Location) at the search moment. We have faced a problem related to the missing data, because participants forget to record some entries. For this reason, we had relied on some experts in the field of Information Retrieval to pick manually the 4600 initial set of queries based on the signification of their terms and the presence of the required information. Those experts are graduate students in the field of Information Retrieval. Also experts have select queries which may be related to the user's environmental and physical context. After a filtering step to eliminate duplicate and navigational queries, we obtained a set of 4000 queries. Where three contextual dimensions (Time, Location and Activity) are assigned to each query to indicate the user current situation.

Search Results and Contextual Dimensions. To obtain the top N Web pages that match each query, we use a web search engine namely Google via the Google Custom Search API ${ }^{1}$. We considered only the first 10 retrieved results, which is reasonable for a mobile browser, because mobile users aren't likely to scroll through long lists of retrieved results.

Then, for each query in the test set we classified manually their related contextual dimensions. Each dimension is associated with a label to indicate whether it is irrelevant or relevant. The criterion to assess whether a given dimension is relevant, is based on whether the mobile user expects to see search results related to this contextual information ordered high on the results list of a search engine. E.g., for a query such as "weather" the user can express his intention to see search results related higher to location and time information. Since, these dimensions are judged relevant.

To classify contextual dimensions, we recruited 10 of our diary study participants (3 male, 7 female). They assign a pertinence degree to each dimension according to their related queries. These steps left us, in our sample test queries, with $10 \%$ noise dimensions, $24 \%$ irrelevant dimensions and $65,6 \%$ relevant dimensions.

\footnotetext{
${ }^{1}$ https://developers.google.com/custom-search/.
} 


\subsection{Classification Performance of Our Metric Measure}

Our experimental design allows us to evaluate the effectiveness of our technique to identify user's relevant contextual fields. For this purpose, we propose an evaluation methodology of obtaining results using manually labelled contextual dimensions. In fact, a contextual dimension's class is correct only if it matches the labelled results. Using the "Preference Score" as a classification feature, we build a context intent classifier.

In order to compute the performance of the classifiers in predicting the dimensions classes, we use standard precision, recall and F-measure measures. We use also classifiers implemented as part of the Weka ${ }^{2}$ software. We test the effectiveness of several supervised individual classifiers (Decision trees, Naive Bayes, SVM, and a Rule-Based Classifier) in classifying contextual fields using "Preference Score" as classification feature.

\subsection{Results and Discussion}

Analysis of Preference Score Measure. At this level we analyse the "Preference Score" distribution for each category of contextual dimensions. Fig. 3 shows the distribution of our measure over different values of Location dimension for different queries. In this figure we notice that there is remarkable drops and peaks in the value of "Preference Score".

Moreover, this distribution of this measure for location dimensions, presented in Fig. 3, has a clear variation with multiple values which clearly support our assumption here. Indeed, the relevance of a contextual dimension is independent of his type or value but it depends on the query and the intention of mobile user behind such query. Hence, "Preference Score" measure hasn't a uniform distribution for those dimensions. It still depends on the user's query. We can conclude that the measure based on the language model approach succeeds to measure the sensitivity of user's query to each contextual dimension.

Table 1 presents the two lowest and two highest values for each dimension class, obtained from our sample test queries and their user's context. Those values allow to confirm a possible correlation between dimension intent class and "Preference Score" feature. Hence, we define a threshold value for each dimension class. And in the following, we will evaluate the effectiveness of thus thresholds to classify those dimensions.

Effectiveness of Contextual Dimension Classification. Our goal in this evaluation is to assess the effectiveness of our classification attribute "Preference Score" to identify the type of dimension of classes: relevant, irrelevant, and noise.

As discussed above, we tested different types of classifiers and Table 2 presents the values of the evaluation metrics obtained by each classifier. In fact, all the classifiers were able to distinguish between the three contextual dimension classes. Fmeasures, Precision and Recall ranging from $96 \%$ to $99 \%$. But "SVM"

\footnotetext{
${ }^{2}$ http://www.cs.waikato.ac.nz/ml/weka/.
} 


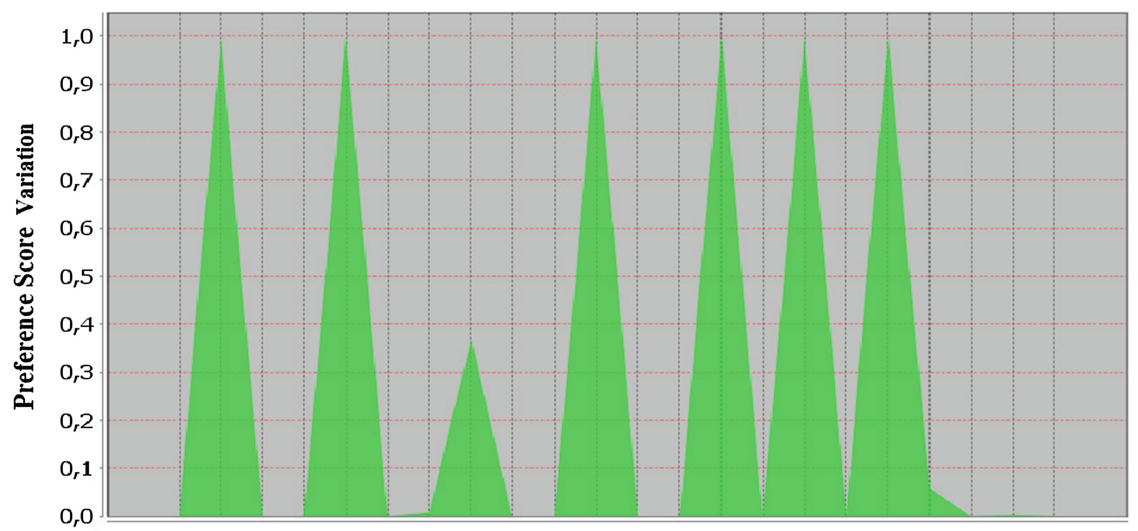

Preference Score measure for Location dimension

Fig. 3. Distribution of "Preference Score" measure for geographic dimension (Location).

classifier achieves the highest accuracy with $99 \%$ for the F-measure. This experiment implies the effectiveness of our approach to accurately distinguish the three types of user's current context levels. It especially allows to correctly identify the irrelevant contextual information with an evaluation measure over 1 . When relevant and noise, achieving over $97 \%$ classification accuracy.

In a second experiment, we evaluated the classification effectiveness of our approach comparatively to the DIR approach developed by Kessler [3]. By using the DIR measure, contextual information is only classified as relevant or irrelevant. It enables distinguishing between irrelevant and relevant context using a threshold value $\delta$. Whence, we compared the two approaches only on this basis.

We implemented the DIR approach using the SVM classifier which achieves one of the best classification performance using one simple rule: analysing the

Table 1. The lowest and highest values for each contextual dimension classes relevant and irrelevant.

\begin{tabular}{l|l|l|l}
\hline Query & Dimensions & Relevance score & Class \\
\hline "house for sale" & Activity: At home & 0.432 & Relevant \\
\hline "check in airport" & Time: 12/09/2012 & 0.550 & Relevant \\
\hline "house for sale" & Location: Tunisia & 0.985 & Relevant \\
\hline "check in airport" & Activity: Walking & 0.999 & Relevant \\
\hline "outdoor tiki hut bars" & Time: 30/08/2012 & 0.147 & Irrelevant \\
\hline "eye chart" & Activity: Working & 0.169 & Irrelevant \\
\hline "weather" & Activity: Walking & 0.199 & Irrelevant \\
\hline "new bus federation" & Activity: Study & 0.279 & Irrelevant \\
\hline
\end{tabular}


Table 2. Classification performance obtained using a classifier with "Preference Score".

\begin{tabular}{|c|c|c|c|c|c|}
\hline Classifier & Class & Precision & Recall & F-measure & Accuracy \\
\hline \multirow[t]{4}{*}{ SVM } & relevant & 0.978 & 0.989 & 0.981 & \multirow[t]{4}{*}{$99 \%$} \\
\hline & irrelevant & 1 & 1 & 1 & \\
\hline & noise & 0.981 & 1 & 0.991 & \\
\hline & average & 0.991 & 0.99 & 0.99 & \\
\hline \multirow[t]{4}{*}{ JRIP rules } & relevant & 0.911 & 0.953 & 0.924 & \multirow[t]{4}{*}{$96.3 \%$} \\
\hline & irrelevant & 1 & 1 & 1 & \\
\hline & noise & 1 & 0.964 & 0.926 & \\
\hline & average & 0.965 & 0.962 & 0.962 & \\
\hline \multirow[t]{4}{*}{ Bayes } & relevant & 1 & 0.933 & 0.966 & \multirow[t]{4}{*}{$97 \%$} \\
\hline & irrelevant & 1 & 1 & 1 & \\
\hline & noise & 0.946 & 1 & 0.972 & \\
\hline & average & 0.973 & 0.971 & 0.971 & \\
\hline \multirow[t]{4}{*}{ J48 } & relevant & 1 & 0.933 & 0.966 & \multirow[t]{4}{*}{$97 \%$} \\
\hline & rrelevant & 1 & 1 & 1 & \\
\hline & noise & 0.946 & 1 & 0.972 & \\
\hline & average & 0.973 & 0.971 & 0.971 & \\
\hline
\end{tabular}

individual results in two rankings for the same query expanded by different contextual dimensions. Intended or relevant contextual information must have an impact that goes beyond a threshold value. Hence, we should obtain a high value of DIR measure to classify a context as relevant. Table 3 presents the precision, recall, F-measure and accuracy achieved by the SVM classifier according to the both approaches. The result of comparison show that, our approach gives higher classification performance than DIR approach with an improvement of $1 \%$ at accuracy. This improvement is mainly over Relevant context dimensions with $1.3 \%$ at Recall.

Table 3. Classification performance on relevant and irrelevant dimensions: comparison between CAP approach and DIR measure approach.

\begin{tabular}{l|l|l|l|l|l|l|l|l|l}
\hline Approach & DIR approach & \multicolumn{2}{l}{ CAP approach } \\
\hline Class & Relevant & Irrelevant & Average & Relevant & Impro & Irrelevant & Impro & Avrege & Impro \\
\hline Precision & 1 & 0.834 & $\mathbf{0 . 9 1 7}$ & 1 & $\mathbf{0 \%}$ & 0.942 & $\mathbf{0 . 1 1 \%}$ & $\mathbf{0 . 9 9 1}$ & $\mathbf{0 . 8 6 \%}$ \\
\hline Recall & 0.874 & 1 & $\mathbf{0 . 9 3 7}$ & 0.934 & $\mathbf{0 . 0 6 \%}$ & 1 & $\mathbf{0 \%}$ & $\mathbf{0 . 9 6 7}$ & $\mathbf{0 . 0 3 \%}$ \\
\hline F-measure & 0.905 & 0.893 & $\mathbf{0 . 8 9 9}$ & 0.981 & $\mathbf{1 . 3 \%}$ & 0.992 & $\mathbf{0 . 9 \%}$ & $\mathbf{0 . 9 9}$ & $\mathbf{1 \%}$ \\
\hline Accuracy & $\mathbf{9 2 \%}$ & & $\mathbf{9 5 \%}$ & & & & $\mathbf{3 \%}$ \\
\hline
\end{tabular}




\section{Conclusion}

We proposed in this paper a new approach for mobile context adaptation to the user's preferences. It evaluates the relevance of contextual dimensions using different features. This approach is based a new metric "Preference Score", that allows to classify the contextual dimensions according to their relevance to enhance the search results. Our experimental evaluation shows the classification performance of our metric measure compared to a cognitively plausible dissimilarity measure namely DIR. For future work, we plan to exploit our proposed approach to personalize mobile Web search. We will customize the search results for queries by considering the determined user's contextual dimension classified as relevant.

\section{References}

1. Abowd, G.D., Dey, A.K.: Towards a better understanding of context and contextawareness. In: Gellersen, H.-W. (ed.) HUC 1999. LNCS, vol. 1707, p. 304. Springer, Heidelberg (1999)

2. Schilit, B., Adams, N., Want, R.: Context-aware computing applications. In: Proceedings of the Workshop on Mobile Computing Systems and Applications, pp. 85-90. IEEE Computer Society, Santa Cruz, CA (1994)

3. Kessler, C.: What is the difference? a cognitive dissimilarity measure for information retrieval result sets. J. Knowl. Inf. Syst. 30(2), 319-340 (2012)

4. Aréchiga, D., Vegas, J., Redondo, P.F.: Ontology supported personalized search for mobile devices. In: Proceedings Third International Workshop on Ontology, Conceptualization and Epistemology for Information Systems, Software Engineering and Service Science, ONTOSE. Springer LNCS, Amsterdam, pp. 1-12, 8-12 June 2009

5. Tsai, F.S., Etoh, M., Xie, X., Lee, W.C., Yang, Q.: Introduction to mobile information retrieval. J. IEEE Intell. Syst. 25(1), 11-15 (2010)

6. Jelinek, F., Mercer, R.L.: Interpolated estimation of markov source parameters from sparse data. In: Proceedings of the Workshop on Pattern Recognition in Practice, pp. 381-397. North-Holland, Amsterdam, The Netherlands, May 1980

7. Castelli, G., Mamei, M., Rosi, A.: The whereabouts diary. In: Hightower, J., Schiele, B., Strang, T. (eds.) LoCA 2007. LNCS, vol. 4718, pp. 175-192. Springer, Heidelberg (2007)

8. Ahn, J., Brusilovsky, J.P., He, D., Grady, J., Li, Q.: Personalized web exploration with task modles. In: Proceedings of WWW 2008 the 17th International Conference on World Wide Web, Beijing, China, pp. 1-10, 21-25 April 2008

9. Hollan, J.D., Sohn, T., Li, K.A., Griswold, W.G.: A diary study of mobile information needs. In: Proceedings of SIGCHI Conference on Human Factors in Computing Systems, pp. 433-442. ACM, Florence, Italy, 5-10 April 2008

10. Pitkow, J., Schutze, H., Cass, T., Cooley, R., Turnbull, D., Edmonds, A., Adar, E., Breuel, T.: Personalized search. Commun. ACM J. 45(9), 50-55 (2002)

11. Ponte, J.M., Croft, W.B.: A language modeling approach to information retrieval. In: Proceedings of the 21st International ACM SIGIR Conference on Research and Development in Information Retrieval, pp. 275-281. ACM, Melbourne, Australia, August 1998 
12. Stefanidis, K., Pitoura, E., Vassiliadis, P.: Adding context to preferences. In: Proceedings of ICDE IEEE 23rd International Conference on Data Engineering, Istanbul, Turkey, pp. 846-855, 15-20 April 2007

13. Gravano, L., Hatzivassiloglou, V., Lichtenstein, R.: Categorizing web queries according to geographical locality. In: Proceedings of CIKM 2003 the Twelfth International Conference on Information and Knowledge Management, pp. 325333. ACM, New Orleans, Louisiana, USA, 2-8 November 2003

14. Arias, M., Cantera, de la Fuente, P., Llamas, C., Vegas, J.: Knowledge-based thesaurus recommender system in mobile web search, In: Proceedings of CERI 1st Spanish Conference on Information Retrieval, Madrid, Spain, 15-16 June 2010

15. Welch, M., Cho, J.: Automatically identifying localizable queries. In: Proceedings of 31st Annual International ACM SIGIR Conference on Research and Development in Information Retrieval, pp. 1185-1186. ACM, Singapore, 20-24 July 2008

16. Kamvar, M., Baluja, S.: A large scale study of wireless search behavior : Google mobile search. In: Proceedings of the SIGCHI 2006 the SIGCHI Conference on Human Factors in Computing Systems, pp. 701-709. ACM, Montreal, Quebec, Canada, 22-27 April 2006

17. Matsuda, N., Hirashima, T., Nomoto, T., Taki, H., Toyoda, J.I.: Context-sensitive filtering for the Web. J. Web Intell. Agent Syst. 1(3), 249-257

18. Bouidghaghen, O., Tamine, L., Boughanem, M.: Context-aware user's interests for personalizing mobile search. In: Proceedings 12th IEEE International Conference on Mobile Data Management, pp. 129-134. IEEE Computer Society, Sweden, 6-9 June 2011

19. Chirita, P., Firan, C., Nejdl, W.: Summarizing local context to personalize global web search. In: Proceedings of CIKM International Conference on Information and Knowledge Management, pp. 287-296. ACM, Arlington, Virginia, USA, 6-11 November 2006

20. Coppola, P., Della Mea, V., Di Gaspero, L., Menegon, D., Mischis, D., Mizzaro, S., Scagnetto, I., Vassena, L.: The context-aware browser. J. IEEE Intell. Syst. 25(1), 38-47 (2010)

21. Ingwersen, P., Jarvelin, K.: The Turn: Integration of Information Seeking and Retrieval in Context, vol. 18, p. 448. Springer, New York (2005)

22. Roman, P.E., Dell, R.F., Velasquez, J.D., Heufeman, P.L.: Identifying user sessions from web server logs with integer programming. J. Intell. Data Anal. 18(1), 43-61

23. McParlane, P.J., Moshfeghi, Y., Jose, J.M.: On contextual photo tag recommendation. In: Proceedings of SIGIR 2013 the 36th International ACM SIGIR Conference on Research and Development in Information Retrieval, pp. 965-968. ACM, Dublin, Ireland, 28 July -01 August 2013

24. Jones, R.: Temporal profiles of queries. J. ACM Trans. Inf. Syst. TOIS 25(3), 14 (2007)

25. De Virgilio, R., Torlone, R.: Modeling heterogeneous context information in adaptive web based applications. In: Proceedings of ICWE 6th International Conference on Web Engineering, pp. 56-63. ACM, Palo Alto, California, USA, 11-14 July 2006

26. Cronen-Townsend, S., Zhou, Y., Croft, W.B.: Predicting query performance. In: Proceedings the 25th Annual International ACM SIGIR Conference on Research and Development in Information Retrieval, pp. 299-306. ACM, Tampere, Finland, 11-15 August 2002

27. Eguchi, S., Copas, J.: Interpreting Kullback Leibler divergence with the NeymanPearson lemma. J. Multivariate Anal. 97, 2034-2040 (2006) 
28. Missaoui, S., Faiz, R.: A new preference based model for relevant dimension identification in contextual mobile search. In: Noubir, G., Raynal, M. (eds.) Networked Systems. LNCS, vol. 8593, pp. 215-229. Springer, Heidelberg (2014)

29. Poslad, S., Laamanen, H., Malaka, R., Nick, A., Buckle, P., Zipf, A.: Crumpet, Creation of user-friendly mobile services personalised for tourism. In: Proceedings of the Second International Conference on 3G Mobile Communication Technologies, Conference Publication No. 477, pp. 28-32. IEEE Computer Society, London, 26-28 March 2001

30. Vadrevu, S., Zhang, Y., Tseng, B., Sun, G., Li, X.: Identifying regional sensitive queries in web search. In: Proceedings of WWW 2008 the 17th International Conference on World Wide Web, pp. 1185-1186, Beijing, China, 21-25 April 2008

31. Yau, S., Liu, H., Huang, D., Yao, Y.: Situation-aware personalized Information retrieval for mobile internet. In: Proceedings of COMPSAC 27th Annual International Computer Software and Applications Conference, pp. 639-644. IEEE Computer Society, Dallas, TX, USA, 3-6 November 2003

32. Gross, T., Klemke, R.: Context modelling for information retrieval: requirements and approaches. IADIS Int. J. WWW/Internet 6(1), 29-42 (2003)

33. Lavrenko, V., Croft, W.B.: Relevance-based language models. In: Proceedings of SIGIR 2001 the 24th Annual International ACM SIGIR Conference on Research and Development in Information Retrieval, pp. 120-127. ACM, New Orleans, Louisiana, USA, 9-13 September 2001

34. Varma, V., Sriharsha, N., Pingali, P.: Personalized web search engine for mobile devices. In: Proceedings of IIIA International Workshop on Intelligent Information Access, Marina Congress Center, Helsinki, Finland, 6-8 July 2006

35. Croft, W.B., Lafferty, J. (eds.): Language Modeling for Information Retrieval. Kluwer Academic Publishers, Dordrecht (2003) 